\title{
Genome-Wide Association Study and QTL Mapping Revealed Genetic Loci for Fusarium Head Blight Resistance in Chinese Hexaploid Wheat
}

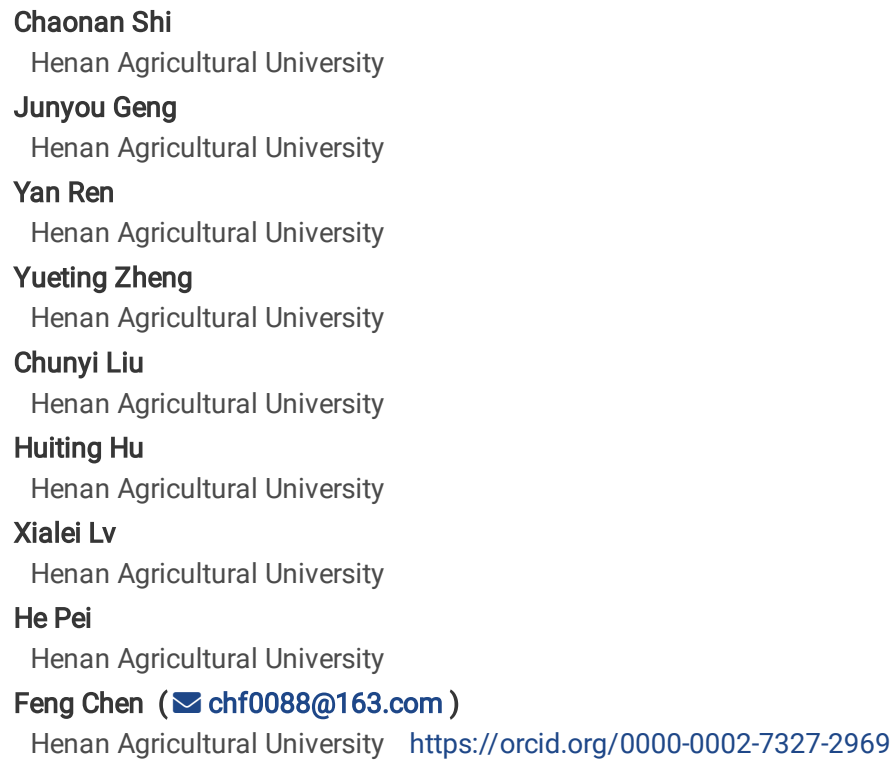




\section{Abstract}

Fusarium Head Blight (FHB) greatly affects wheat yield worldwide and could also downgrade quality. In this study, a total of 406 wheat cultivars were investigated for FHB disease index (DI). Results indicated that the FHB DI of 95\% of the tested cultivars showed more than scale 2 . Genome-wide association study (GWAS) identified 321 SNPs significantly related to FHB resistance on all chromosomes with $2.71-13.32 \%$ phenotypic variance explained (PVE). An important genetic locus $g F H B-5 A(329,828,930$ to $595,372,995 \mathrm{bp})$ was identified to modulate FHB resistance in two panels, and the incidence of resistant alleles at the $g F H B-5 A 1 a$ locus were increasing with time in the tested cultivars. Two loci of $g F H B-2 B(9,740,162$ to $68,200,954$ bp) with PVE values of 2.73$3.57 \%$ and $g F H B-7 A(515,126,041$ to $537,557,064$ bp) with PVE values of $2.71-7.04 \%$ were identified to be significantly associated with FHB resistance. Linkage analysis in a bi-parental population identified the QTL FHB.hau-4D flanked by markers wPt-8836 and wPt-4572 with PVE value of $8.9 \%$. This study identified new genetic loci to control FHB resistance and provided useful information of marker-assist selection in wheat FHB resistance breeding.

\section{Introduction}

Fusarium head blight (FHB), mainly caused by Fusarium graminearum (syn. Gibberella zeae), is an important fungal disease of wheat (Triticum aestivum L.) (Desjardins \& Hohn, 1997; McMullen et al., 2012). Wheat infected by the pathogen showed shriveled kernels with low weight, and red episperm and endosperm. F. graminearum generally produce several kinds of mycotoxins, such as deoxynivalenol (DON) and other trichothecene toxins. Infected wheat kernels with excessive accumulation of DON pose a severe threat to human and animal health (Cheng et al., 2012; Nganji et al., 2004).

It is well known that breeding resistant cultivars is one of the most economical and effective methods to manage disease, therefore it is particularly important to identify and develop FHB-resistant germplasm by combination of molecular marker-assisted selection with backcross selection and multi-point identification (Bai et al., 2012; Lan et al., 2009; Lan et al., 2010a; Lan et al., 2010b; Lu et al., 2019). Previously, two Chinese early historical accessions 'Sumai 3' and 'Wangshuibai' have been identified as highly resistant to FHB (Bai et al., 1999; Chen et al., 1998). Up to date, a large number of FHB resistance QTL has been reported on all wheat chromosomes and molecular markers have been developed for most of the mapped QTL (Buerstmayr et al., 2002; Buerstmayr et al., 2003; Steiner et al., 2017). Among them, a major gene Fhb1 in Sumai 3 was widely reported (Anderson et al., 2001; Bai et al., 1999; Buerstmayr et al., 2002; Cuthbert et al., 2006; Lv et al., 2014). With the development of molecular markers, Fhb1 was further fine mapped to a 1.1-Mb region (Schweiger et al., 2016). Through map-based cloning, a pore-forming toxin-like (PFT) gene encoding a chimeric lectin was regarded as Fhb1 to modulate FHB resistance (Rawat et al., 2016). However, two independent studies showed that TaHRC encoding a putative histidine-rich calcium-binding protein was the key gene within the interval of Fhb1 locus to regulate FHB resistance (Li et al., 2019; Su et al., 2019). Recently, a Fhb7 gene from wheatgrass encoding GST protein showed a new solution for FHB resistance (Wang et al. 2020). It suggests that identification of new loci that confers FHB resistance is necessary in order to pyramid several loci/genes for developing FHB-resistant germplasm.

Common wheat $(2 n=42)$ is an allohexaploid (AABBDD) with a large genome size ( $16 \mathrm{~Gb})$ and high sequence duplication between subgenomes as well as abundance of repetitive elements (about $85 \%$ of the genome) (IWGSC, 2014). With the rapid development of sequencing technologies in recent years, the wheat genome has been sequenced and provides a solid platform for cloning genes in wheat. The development of wheat high-density SNP arrays (Sun et al. 2020) including the Wheat 90K iSelect assay (Wang et al., 2014), 660K (Zhou et al., 2018), and 35K Wheat Breeder's assay (Allen et al., 2017) made it feasible to rapidly excavate genetic loci underlying complex traits in the whole wheat genome by genome-wide association studies (GWAS). In hexaploid wheat, GWAS has been widely used to identify many important genetic loci for regulating kinds of traits, including black point (Liu et al., 2017; Lv et al. 2020), herbicide resistance (Shi et al. 2020), Fusarium crown rot (Yang et al., 2019), and quality traits (Chen et al., 2019), etc.

In the present study, Chinese cultivars mainly from the Huanghuai valley and a bi-parental population were used to identify genetic loci of FHB resistance by GWAS and linkage mapping. These results could provide valuable information for marker-assisted selection through pyramiding more FHB -resistance loci in wheat breeding programs.

\section{Materials And Methods}

\section{Plant materials}

A total of 406 Chinese wheat accessions were divided into two panels in this study. Panel I contained 243 wheat cultivars or advanced lines from the Huanghuai valley, the most important and largest wheat production area with 60 to $70 \%$ of both total harvested area and total wheat production in China, and they all were developed after 2014 as we previously described in Yang et al., (2019) (Table S1). Panel II contained 163 Chinese landraces and historical wheat cultivars (Chen et al., 2019; Sun et al., 2017) (Table S1). A bi-parental recombinant inbred line (RIL) population named UP derived from a cross between the susceptible parent UC1110 (DI=3) and the resistant parent PI610750 (DI=1) (Lowe et al., 2011) with 187 lines was used for QTL mapping. This RIL population was kindly provided by Jorge Dubcovsky from UC Davis. All the test accessions were planted in Jianyang (N27.30 ${ }^{\circ}$, E118.2 ${ }^{\circ}$ ) in $2016-2017$ cropping season with two replications, and 'Xinmai 18 ' as a susceptible control was planted every 10 accessions.

\section{Evaluation of wheat FHB index}

Identification of FHB resistance was carried out at the anthesis stage, and disease symptoms were recorded on the 20th and 30th after the first spray inoculation, fllowing the inoculation method of Steiner et al. (2004). A 1-4 scale was used to represent different disease index (DI) according to the severity of the FHB of the whole line (Feng, 2018). An average DI of 10 spikes was used for each test variety, where

$1=$ infected spikelets account for $1 / 4$ of the total;

$2=$ infected spikelets account for $1 / 2$ of the total; 
$3=$ infected spikelets account for $3 / 4$ of the total;

$4=$ infected spikelets account for more than $3 / 4$ of the total or the whole ear died.

\section{Genotyping and genome-wide association study}

and Panel II were genotyped using the wheat 660K (Yang et al., 2019) and 90K SNP arrays (Wang et al., 2014), respectively. Quality control was performed for SNP markers with MAF $>0.05$ and missing data $<20 \%$. This pre-processing was examined by PLINK software (Purcell et al., 2007).

GWAS was performed based on the mixed linear model (PCA+K) using GAPIT packages in $R$ software (Lipka et al., 2012; Lv et al., 2020; the R Core Team, 2019). The corrected $P$-values of $1.0 \mathrm{e}-3$ and $3.16 \mathrm{e}-3$ were used as the appropriate significance threshold for and II, respectively. Haplotype analysis was performed using Haploview 4.2 software (Barrett et al., 2005).

\section{QTL mapping}

The bi-parental RIL population UP was previously constructed by Lowe et al. (2011) and the genetic linkage map was composed of 1,494 probes (DArT, SSR, and ESTs). Based on polymorphism, 558 markers were extracted for QTL mapping. Genetic linkage map was constructed using IciMapping V4.1

(http://www.isbreeding.net/), and measurement of linkage algorithm and criteria were completed by nnTwoOpt and SAD (Li et al., 2011), and Kosambi was used for genetic function. Phenotypic variance explained (PVE) was used to evaluate QTL effect (Ren et al., 2017) based on ICIM-ADD model. Default was used for parameter designs. LOD scores for detecting significant QTL were calculated at the $P=0.05$ level from 1,000 permutations, and the threshold of LOD score was set to 2.5 .

\section{Statistical analysis}

SPSS ver. 20.0 software and least significant range (LSR) multiple comparisons were used to calculate average DI of cultivars with different haplotypes and compare their significant difference.

\section{Results}

\section{FHB resistance distribution in Chinese wheat cultivars}

Investigation of FHB in 406 wheat cultivars indicated that the mean disease index (DI) of and II was 2.47 ( \pm 0.52$)$ and 2.33 ( \pm 0.59$)$ (Fig. 1a), and more than $95 \%$ wheat cultivars showed FHB DI of more than 2 (Fig. 1b). In Panel I, 0.5\%, 37\%, 57\% and 5.5\% of the accessions showed FHB DI of 0 to 1,1 to 2,2 to 3 , and 3 to 4, respectively. In Panel II, 4\%, 45\%, 45\%, and 6\% of them showed FHB DI of 0 to 1,1 to 2,2 to 3 , and 3 to 4 , respectively. The correlation coefficients between the two replicates in Panel I and Panel II are 0.73 and 0.68 , respectively. Results showed that cultivars with FHB DI of 1 to 3 were predominant, and there were small number of accessions with good FHB resistance. For example, 8 cultivars (Shengyuan 928, Jimai 36, Jimai 32, Youmai 2, Yunong 201, Changnong 339-5-1, Dan 98-4025, and Beijing 841) showed FHB DI of 1 in multiple replications, and they could be used as FHB-resistant germplasms in wheat breeding.

\section{Genome-wide association study of wheat FHB resistance}

After filtering, a total of 395,782 SNPs in Panel I and 41,561 SNPs in Panel II were used for GWAS analysis (Fig. 2). A total of 321 significant SNPs with PVE of $2.71 \%$ to $13.32 \%$ were detected and they mainly distributed on chromosomes $2 \mathrm{~B}, 5 \mathrm{~A}$, and $7 \mathrm{~A}$ (Table S2).

In Panel I, 204 SNPs with PVE of $2.71 \%$ to $9.76 \%$ were significant in two replications. Among them, 55, 24, and 49 significant SNPs mainly distributed on 2B (9,740,162 to $68,200,954$ bp), 5A (329,828,930 to 595,372,995 bp), and 7A (515,126,041 to 537,557,064 bp) (Fig. 2a; Fig. 3a; Table S1). In Panel II, 117 SNPs were significant in two replications with the PVE of $5.74 \%$ to $13.32 \%$, and 36 of them mainly distributed on 5 A (329,828,930 to 595,372,995 bp) (Fig. 2; Fig. $3 \mathrm{~b}$; Table S1). Combination of Panels I and II showed that there was an important genetic locus on 5 A named as $g F H B-5 A(329,828,930$ to $595,372,995$ bp) for FHB resistance in two panels, and 60 significant SNPs located in this interval (Table 1; Table S1). Furthermore, gFHB-2B (9,740,162 to 68,200,954 bp) and gFHB-7A (515,126,041 to 537,557,064 bp) were also the important FHB resistance loci in Panel I since there were 55 and 49 significant SNPs in intervals of gFHB-2B and $g F H B-7 A$, respectively (Fig. 2a; Table S1). In addition, some other FHB resistance loci on 3A (1,326,004 to 12,813,934 bp), 3D (2,597,023 to $92,375,516), 5 \mathrm{D}(426,001,273$ to $544,697,840 \mathrm{bp})$, and $7 \mathrm{~B}(675,149,692$ to $714,012,248)$ were also detected in two panels.

\section{Haplotype analysis of $g F H B-5 A$ in Chinese cultivars}

Haplotype analysis (Fig. 4a, Table 2) in Panel I showed that 21 significant SNPs on 5A (577,926,236 to 588,752,395 bp) developed a 10.83-Mb block (PI_Hap_5A7) with two haplotypes (Table 2). Phenotypic comparison indicated that cultivars with PI_Hap_5A1a (2.37 to 2.4) showed a stronger FHB resistance than those with PI_Hap_5A1b (2.75 to 3.45) (Fig. 4c). In Panel II, 17 significant SNPs on 5A (331,401,559 to 422,077,781 bp) formed a 90.68-Mb block (PII_Hap_5A1) and 7 significant SNPs (591,155,558 to 595,372,995 bp) on 5A formed a 4.22-Mb block (PII_Hap_5A2) (Fig. 4b). PII_Hap_5A1 contained two haplotypes (Table 2). Phenotypic comparison indicated that cultivars with PII_Hap_5A1a (2 to 2.24) showed a stronger FHB resistance than those with PII_Hap_5A1b (2.55 to 2.64) (Fig. 4d). PII_Hap_5A2 contained two haplotypes, and phenotypic comparison indicated that cultivars with PII_Hap_5A2a (2.06 to 2.1) showed a stronger FHB resistance than those with PII_Hap_5A2b (2.5 to 2.52) (Fig. 4e). Therefore, cultivars with PI_Hap_5Aa, PII_Hap_5A1a, and PII_Hap_5A2a showed relatively stronger resistance to FHB. Pyramiding analysis indicated that cultivars with PII_Hap_5A1a/PII_Hap_5A2a (1.7 to 2) showed significantly stronger FHB disease resistance than those with PII_Hap_5A1b/PII_Hap_5A2b (2.8 to 2.9) in Panel II (Fig. 4f, Table 2). PII_Hap_5A1a, PII_Hap_5A1a, and PII_Hap_5A2a could be used for marker-assisted selection in FHB resistance breeding. 
All cultivars from Panel I were developed after 2014, and 67.5\% of them possessed FHB-resistant allele PI_Hap_5A1a, indicating that it has been widely used in the Huanghuai valley (Fig. 5a). Based on released years of cultivars, Panel II could be divided into three periods, i.e. before 1980 s, 1980 s to 2000 s, and after 2000s. Percentages of FHB-resistant allele PII_Hap_5A1a are $11.1 \%, 11.4 \%$, and $14.8 \%$ in the cultivars of before 1980 s, 1980 s to 2000 s, and after 2000 s, respectively (Fig. 5b). Moreover, percentages of FHB-resistant PII_Hap_5A2a are $11.1 \%, 16.5 \%$, and $22.2 \%$ in the cultivars of before 1980 s, 1980 s to 2000 s, and after 2000s, respectively (Fig. 5b). It suggested that FHB-resistant alleles at the $g F H B-5 A$ locus were becoming prevalent with time in Chinese cultivars.

\section{Linkage mapping for FHB resistance in UP population}

Linkage analysis in the RIL population UP showed that a QTL (FHB.hau-4D) on chromosome 4D was detected for FHB resistance and it was flanked by markers wPt-8836 and wPt-4572 with PVE of $8.89 \%$. Wheat lines with FHB.hau-4D_AA (2.37) showed a stronger FHB resistance than those with FHB.hau$4 D \_B B(2.73)$ (Fig. 6). Analysis of physical position in the Chinese Spring genome database indicated that FHB.hau-4D was mapped to be around $9431967-$ bp position on $4 \mathrm{D}$.

\section{Discussion}

As one of most serious diseases that threaten wheat yield and quality, FHB has been widely studied for screening resistance germplasm and QTL mapping. Among all of the FHB-resistant QTL detected in wheat and its relative, Fhb1-Fhb7 have formally been designated as the important genetic loci or genes. Of all 7 loci, both of Fhb1 on 3BS and Fhb2 on 6B were derived from 'Sumai 3' (Cuthbert et al., 2006; Cuthbert et al., 2007), and Fhb4 is from 'Wangshuibai' (Xue et al., 2010), and Fhb5 on 5A is from 'Wangshuibai' and 'Sumai 3' (Xue et al., 2011), and both Fhb6 and Fhb7 are from wild relatives of wheat (i.e. Leymus racemosus, Elymus tsukushiensis and Thinopyrum ponticum) (Cainong et al., 2015; Guo et al., 2015; Qi et al., 2008).

Fhb1 locus on 3BS as the most stable and largest effect QTL was first reported in Sumai3 and its derivative Ning7840 (Bai et al., $1999 ;$ Rawat et al., 2016 ; Waldron et al., 1999). Fhb1 gene was first reported to be a pore-forming toxin-like (PFT) gene encoding a chimeric lectin with two agglutinin domains and an ETX/MTX2 toxin domain (Rawat et al., 2016). But more recently, two different groups simultaneously reported a new gene TaHRC as Fhb1, and it encodes a putative histidine-rich calcium-binding protein. TaHRC conferred FHB susceptibility and the deletion spanning its start codon can result in FHB resistance (Li et al., 2019; Su et al., 2019). Identification of candidate genes at other loci are still ongoing. Here, 10 significant SNPs were identified on 3B by GWAS, and Fhb1 gene and Qfhb.hwwg-3BSc (Somers et al.,, 2003) were in the interval we identified in the present study.

The genetic locus on $5 \mathrm{~A}$ ( $\mathrm{gFHB}-5 \mathrm{~A})$ in the present study was identified in both Panels I and II. This locus contained 60 significant SNPs in the region of $329,828,930$ to $595,372,995$ bp on 5 A. Compared to previous studies, Fhb5 on 5A was the important resistance locus from Wangshuibai and Sumai 3 (Brar et al., 2019; Lin et al., 2006). Steiner et al. (2019) identified two QTL on 5A in Sumai3. The major QTL Qfhs.ifa-5Ac was mapped to be close to centromere and the smaller QTL Qfhs.ifa-5AS(70.7-195.3 Mb) was mapped to the distal of chromosome 5AS with distance of $140 \mathrm{Mb}$ from Qfhs.ifa-5Ac (245.9-290 Mb). However, Zhang et al. (2012) identified that Qfhb.hwwg-5A was flanked with Xbarc141and Xgwm186 (468.9-471.7 Mb) in Baishanyuehuang, and Qfhb.hwwg$5 A$ were also mapped between Xgwm129 and Xbarc197 (492,893,704 to 492,893,948 bp) in Frontana and QFhbp-jaas.5AL-1 was linked to BS00069175_51 (485,598,111 to $485,598,143$ bp) (Steiner et al., 2004; Yi et al., 2018). Therefore, there is at least one important genetic locus on $5 \mathrm{~A}$ to control FHB resistance. The $g F H B-5 A$ we identified in the present study is possibly the same with the $Q f h b$. $h w w g-5 A$ or $Q F h b p-j a a s .5 A L-1$ though they are from different wheat cultivars.

In Panel I, FHB-resistant loci on 2B and 7A were identified by GWAS. On 2B, significant SNPs mainly clustered into 9,740,162 to 68,200,954 bp with PVE of 2.71-4.17\%, but it was only detected in Panel I. QTL on 2B was previously reported in different wheat accessions containing Ning7840 (Zhou et al., 2002) and Ernie (Liu et al., 2007). A QTL on 2BS was closely linked to Xgwm210 in Renan and in Patterson/Goldfield population (Gervais et al., 2003; Gilsinger et al., 2005). The QTL Qfhb.nc-2B.1 flanking with IWB31987 and IWB40514 (46.5-68.2 Mb) was identified in the Truman/MO 94-317 population, and this QTL was possibly the same with the $g F H B-2 B$ we identified in the present study. On 7A, significant SNPs mainly clustered in the interval of $515,126,041$ to $537,557,064$ bp, and it has long physical distance from the previous QTL (XsdauK66 Xcfa2240) on 7A identified in Thinopyrum ponticum. Moreover, FHB.hau-4D (wPt8836 wPt-4572) was identified by linkage mapping in UP population. Previous studies identified some FHB-resistant QTL on chromosome 4D in the population of ChineseSpring/SM3-7ADS and Spark/Arina (Draeger et al., 2007; Ma et al., 2006; Srinivasachary et al., 2008) but it need more work to confirm if they are the same locus. Although some resistance loci were excavated in this study, some loci were not very stable since they were only significant in one replicates possibly because of environmental influence. However, one stable resistance loci identified in this study could be considered to use for makerassisted selection in improvement of FHB resistance in common wheat.

\section{Abbreviations}

FHB: Fusarium Head Blight

GWAS: Genome wide association study

DI: Disease index

SNP: Single nucleotide polymorphisms

PVE: Phenotypic variance explained 
DON: Deoxynivalenol

PFT: Pore-forming toxin-like

MAF: Minor allele frequency

QTL: Quantitative trait locus

RIL: Recombinant inbred line

\section{Declarations}

\section{Funding}

This work was funded by the National Key Research and Development Program (2019YFE0118300), the National Natural Science Foundation (31861143008 and U1904109), Henan Science and Technology Projects (202102110016 and 201300110800) of China.

\section{Author Contributions}

FC conceived and designed the experiments; CS, JG, YR, YZ, CL, HH, XL, and HP performed phenotype observation, GWAS analysis and QTL mapping; CS and FC wrote the paper.

\section{Competing interests}

The authors declare that they have no competing interests.

Data Availability Statement

Not applicable.

\section{Ethics approval and consent to participate}

Not applicable.

\section{Consent for publication}

\section{References}

1. Allen AM, Winfield MO, Burridge AJ et al (2017) Characterization of a wheat breeders' array suitable for high-throughput SNP genotyping of global accessions of hexaploid bread wheat (Triticum aestivum). Plant Biotechnol Journal 15:390-401

2. Anderson JA, Stack RW, Liu S, et al., (2001) DNA markers for Fusarium head blight resistance QTLs in two wheat populations. Theor Appl Genet 102:1164-1168

3. Bai B, He ZH, Asad MA et al (2012) Pyramiding adult-plant powdery mildew resistance QTLs in bread wheat. Crop Pasture Science 63:609-611

4. Bai G, Kolb FL, Shaner G, Domier LL (1999) Amplified fragment length polymorphism markers linked to a major quantitative trait locus controlling scab resistance in wheat. Phytopathology 89:343-348

5. Barrett JC, Fry B, Maller J, Daly MJ (2005) Haploview: analysis and visualization of LD and haplotype maps. Bioinformatics 21:263-265

6. Brar GS, Brûlé-Babel AL, Ruan Y, et al., (2019) Genetic factors affecting Fusarium head blight resistance improvement from introgression of exotic Sumai 3 alleles (including Fhb1, Fhb2, and Fhb5) in hard red spring wheat. BMC Plant Biology 19, 179

7. Buerstmayr H, Lemmens M, Hartl L, et al., (2002) Molecular mapping of QTLs for Fusarium head blight resistance in spring wheat. I. Resistance to fungal spread (Type II resistance). Theor Appl Genet 104:84-91

8. Buerstmayr H, Steiner B, Hartl L, et al., (2003) Molecular mapping of QTLs for Fusarium head blight resistance in spring wheat. II. Resistance to fungal penetration and spread. Theor Appl Genet 107:503-508

9. Cainong JC, Bockus WW, Feng Y et al (2015) Chromosome engineering, mapping, and transferring of resistance to Fusarium head blight disease from Elymus tsukushiensis into wheat. Theor Appl Genet 128:1019-1027

10. Chen J, Zhang F, Zhao C et al (2019) Genome-wide association study of six quality traits reveals the association of the TaRPP13L 1 gene with flour colour in Chinese bread wheat. Plant Biotechnol J 17:2106-2122

11. Chen P, Sun W, Liu W et al., 1998. Development of wheat Leymus racemosus translocation lines with scab resistance. Proceedings of the 9th international wheat Genetics symposium. University Extension Press, Saskatoon, Canada. 32-34

12. Cheng SH, Zhang Y, Bie TD et al (2012) Damage of wheat Fusarium head blight epidemics and geneticimprovement of wheat for scab resistance in China. Jiangsu Journal of Agricultural Science 28:938-942

13. Cuthbert PA, Somers DJ, Thomas J, Cloutier S, Brûlé-Babel A (2006) Fine mapping Fhb1, a major gene controlling Fusarium head blight resistance in bread wheat (Triticum aestivum L.). Theor Appl Genet 112:1465-1702 
14. Cuthbert PA, Somers DJ, Brûlé-Babel A (2007) Mapping of Fhb2 on chromosome 6BS: a gene controlling Fusarium head blight field resistance in bread wheat (Triticum aestivum L.). Theor Appl Genet 114:429-437

15. Desjardins AE, Hohn TM (1997) Mycotoxins in plant pathogenesis. Mol Plant Microbe Interact 10:147-152

16. Draeger R, Gosman N, Steed A et al (2007) Identification of QTLs for resistance to Fusarium head blight, DON accumulation and associated traits in the winter wheat variety Arina. Theor Appl Genet 115:617-625

17. Feng J (2018) Creation of wheat cultivars for resistance to Fusarium head blight and analysis of resistance sources. Northwest A\&F University, May

18. Gervais L, Dedryver F, Morlais JY et al (2003) Mapping of quantitative trait loci for Weld resistance to Fusarium head blight in an European winter wheat. Theor Appl Genet 106:961-970

19. Gilsinger J, Kong L, Shen X, Ohm H (2005) DNA markers associated with low Fusarium head blight incidence and narrow flower opening in wheat. Theor Appl Genet 110:1218-1225

20. Guo J, Zhang X, Hou Y et al (2015) High-density mapping of the major FHB resistance gene Fhb7 derived from Thinopyrum ponticum and its pyramiding with Fhb1 by marker-assisted selection. Theor Appl Genet 128:2301-2316

21. International Wheat Genome Sequencing Consortium (IWGSC) (2014) A chromosome-based draft sequence of the hexaploid bread wheat (Triticum aestivum) genome. Science 345:1251788

22. Lan CX, Liang SS, Wang ZL et al (2009) Quantitative trait loci mapping for adult-plant resistance to powdery mildew in Chinese wheat cultivar Bainong 64. Phytopathology 99:1121-1126

23. Lan CX, Liang SS, Zhou XC et al (2010a) Identification of genomic regions controlling adult-plant stripe rust resistance in Chinese Landrace Pingyuan 50 through bulked segregant analysis. Phytopathology 100:313-318

24. Lan CX, Ni XW, Yan J et al (2010b) Quantitative trait loci mapping of adult-plant resistance to powdery mildew in Chinese wheat cultivar Lumai 21. Mol Breeding 25:615-622

25. Li HH, Bradbury P, Ersoz E, Buckler ES, Wang JK (2011) Joint QTL linkage mapping for multiple-cross mating design sharing one common parent. PLoS One 6:e17573

26. Li G, Zhou J, Jia H et al (2019) Mutation of a histidine-rich calcium-binding-protein gene in wheat confers resistance to Fusarium head blight. Nat Genet $51: 1106-1112$

27. Lin F, Xue SL, Zhang ZZ et al (2006) Mapping QTL associated with resistance to Fusarium head blight in the Nanda2419 x Wangshuibai population. II: Type I resistance. Theor Appl Genet 112:528-535

28. Lipka AE, Tian F, Wang Q, et al., (2012) GAPIT: genome association and prediction integrated tool. Bioinformatics 28:2397-2399

29. Liu J, He Z, Rasheed A et al (2017) Genome-wide association mapping of black point reaction in common wheat (Triticum aestivum L.). BMC Plant Biol 17:220

30. Liu S, Abate ZA, Lu H, Muske T, Davis GL, McKendry AL (2007) QTL associated with Fusarium head blight resistance in the soft red winter wheat Ernie. Theor Appl Genet 115:417-427

31. Lowe I, Jankuloski L, Chao S, Chen X, See D, Dubcovsky J (2011) Mapping and validation of QTL which confer partial resistance to broadly virulent post2000 North American races of stripe rust in hexaploid wheat. Theor Appl Genet 123:143-157

32. Lu YM, Lan CX, Liang SS et al (2009) QTL mapping for adult-plant resistance to stripe rust in Italian common wheat cultivars Libellula and Strampelli. Theor Appl Genet 119:1349-1359

33. Lv C, Song Y, Gao L et al., 2014. Integration of QTL detection and marker assisted selection for improving resistance to Fusarium head blight and important agronomic traits in wheat. Crop Journal $70-78$

34. Lv G, Dong Z, Wang Y et al., 2020. Identification of genetic loci of black point in Chinese common wheat by genome-wide association study and linkage mapping. Plant Disease In press

35. Ma H, Bai G, Gill BS, Hart LP (2006) Deletion of a Chromosome Arm Altered Wheat Resistance to Fusarium Head Blight and Deoxynivalenol Accumulation in Chinese Spring. Plant Dis 90:1545-1549

36. McMullen M, Bergstrom G, De-Wolf E et al (2012) A Unified Effort to Fight an Enemy of Wheat and Barley: Fusarium Head Blight. Plant Dis $96: 1712$

37. Nganje WE, Bangsund DA, Leistritz FL et al (2004) Regional economic impacts of Fusarium head blight in wheat and barley. Review of agricultural economics 26:332-347

38. Purcell S, Neale B, Todd-Brown K et al (2007) Plink: a tool set for whole-genome association and population-based linkage analyses. Am J Hum Genet 81:559-575

39. Qi L, Pumphrey M, Friebe B et al (2008) Molecular cytogenetic characterization of alien introgressions with gene Fhb3 for resistance to Fusarium head blight disease of wheat. Theor Appl Genet 117:1155-1166

40. Rawat N, Pumphrey MO, Liu S et al (2016) Wheat fhb1 encodes a chimeric lectin with agglutinin domains and a pore-forming toxin-like domain conferring resistance to Fusarium head blight. Nat Genet 48:1576-1580

41. Ren Y, Hou W, Lan C et al (2017) QTL Analysis and Nested Association Mapping for Adult Plant Resistance to Powdery Mildew in Two Bread Wheat Populations. Front Plant Sci 8:1212

42. Schweiger W, Steiner B, Vautrin S et al (2016) Suppressed recombination and unique candidate genes in the divergent haplotype encoding Fhb1. Theor Appl Genet 129:1607-1623 
43. Somers DJ, Fedak G, Savard M (2003) Molecular mapping of novel genes controlling Fusarium head blight resistance and deoxynivalenol accumulation in spring wheat. Genome 46:555-564

44. Srinivasachary GN, Steed A et al (2008) Susceptibility to Fusarium head blight is associated with the Rht-D1b semi-dwarfing allele in wheat. Theor Appl Genet 116:1145-1145

45. Steiner B, Griesser M, Scholz U, Schondelmaier J, Buerstmayr H (2004) Molecular mapping of resistance to Fusarium head blight in the spring wheat cultivar Frontana. Theor Appl Genet 109:215-224

46. Steiner B, Buerstmayr M, Michel S et al (2017) Breeding strategies and advances in line selection for Fusarium head blight resistance in wheat. Tropical Plant Pathology 42:165-174

47. Steiner B, Buerstmayr M, Wagner C et al (2019) Fine-mapping of the Fusarium head blight resistance QTL Qfhs.ifa-5A identifies two resistance QTL associated with anther extrusion. Theor Appl Genet 132:2039-2053

48. Su Z, Bernardo A, Tian B et al (2019) A deletion mutation in TaHRC confers Fhb1 resistance to Fusarium head blight in wheat. Nat Genet 51:1099-1105

49. Sun C, Dong Z, Zhao L et al (2020) The Wheat 660K SNP array demonstrates great potential for marker-assisted selection in polyploid wheat. Plant Biotechnol J 18:1354-1360

50. Sun C, Zhang F, Yan X et al (2017) Genome-wide association study for 13 agronomic traits reveals distribution of superior alleles in bread wheat from the Yellow and Huai Valley of China. Plant Biotechnol J 15:953-969

51. Waldron B, Moreno-Sevilla B, Anderson J, Stack R, Frohberg R (1999) RFLP mapping of QTL for Fusarium head blight resistance in wheat. Crop Sci 39:805-811

52. Wang HW, Sun SL, Ge WY et al (2020) Horizontal gene transfer of Fhb7from fungus underlies Fusarium head blight resistance in wheat. Science 22(6493):eaba5435 368(

53. Wang S, Wong D, Forrest K et al (2014) Characterization of polyploid wheat genomic diversity using the high-density 90,000 SNP array. Plant Biotechnol J 12:787-796

54. Xue S, Li G, Jia H et al (2010) Fine mapping Fhb4, a major QTL conditioning resistance to Fusarium infection in bread wheat (Triticum aestivum L.). Theor Appl Genet 121:147-156

55. Xue S, Xu F, Tang M et al (2011) Precise mapping Fhb5, a major QTL conditioning resistance to Fusarium infection in bread wheat (Triticum aestivum L.). Theor Appl Genet 123:1055-1063

56. Yang X, Pan Y, Singh PK et al (2019) Investigation and genome-wide association study for Fusarium crown rot resistance in Chinese common wheat. BMC Plant Biol 19:153

57. Yi X, Cheng J, Jiang Z et al (2018) Genetic Analysis of Fusarium Head Blight Resistance in CIMMYT Bread Wheat Line C615 Using Traditional and Conditional QTL Mapping. Front Plant Sci 9:573

58. Zhang X, Pan H, Bai G (2012) Quantitative trait loci responsible for Fusarium head blight resistance in Chinese landrace Baishanyuehuang. Theoretical Applied Genetics Aug 125(3):495-502

59. Zhou S, Zhang J, Che Y et al (2018) Construction of Agropyron Gaertn. genetic linkage maps using a wheat 660K SNP array reveals a homoeologous relationship with the wheat genome. Plant Biotechnol J 16:818-827

60. Zhou WC, Kolb FL, Bai GH, Shaner G, Domier LL (2002) Genetic analysis of scab resistance QTL in wheat with microsatellite and AFLP markers. Genome Aug 45(4):719-727

\section{Tables}

Table 1 FHB resistance loci identified by GWAS in two panels. 


\begin{tabular}{|c|c|c|c|c|c|c|c|c|}
\hline \multirow[t]{2}{*}{ Chrom. } & \multicolumn{2}{|l|}{ Left marker } & \multicolumn{2}{|l|}{ Right marker } & \multirow{2}{*}{$\begin{array}{l}\text { Range } \\
(\mathrm{Mb})\end{array}$} & \multirow{2}{*}{$\begin{array}{l}\text { Number of } \\
\text { interval/total } \\
\text { significant } \\
\text { SNPs }\end{array}$} & \multirow{2}{*}{$\begin{array}{l}\text { PVE } \\
(\%)\end{array}$} & \multirow{2}{*}{$\begin{array}{l}\text { Panel and } \\
\text { replicate }\end{array}$} \\
\hline & Name & Position & Name & Position & & & & \\
\hline $1 \mathrm{~A}$ & AX-110577325 & 2936928 & AX-110544824 & 63341634 & 60.40 & $32 / 35$ & $\begin{array}{l}2.74- \\
9.02\end{array}$ & $\begin{array}{l}\text { Panel_I_R2, } \\
\text { Panel_II_R1 }\end{array}$ \\
\hline 1B & Kukri_c51864_225 & 582906917 & BS00076330_51 & 626724382 & 43.82 & $4 / 6$ & $\begin{array}{l}5.79- \\
10.00\end{array}$ & $\begin{array}{l}\text { Panel_II_R1, } \\
\text { Panel_II_R2 }\end{array}$ \\
\hline 1D & AX-95008362 & 228239 & AX-109306242 & 5444062 & 5.22 & $6 / 7$ & $\begin{array}{l}2.74- \\
3.44\end{array}$ & Panel_I_R2 \\
\hline $2 \mathrm{~A}$ & AX-111074099 & 703111613 & AX-111137145 & 703559811 & 0.45 & $10 / 11$ & $\begin{array}{l}7.04- \\
9.36\end{array}$ & Panel_I_R1 \\
\hline $2 \mathrm{~B}$ & AX-109423210 & 9740162 & AX-89562766 & 68200954 & 58.46 & $55 / 60$ & $\begin{array}{l}2.71- \\
4.17\end{array}$ & Panel_I_R2 \\
\hline $2 \mathrm{D}$ & D_contig17313_245 & 9344681 & Kukri_c27335_619 & 35053295 & 25.71 & $3 / 7$ & $\begin{array}{l}8.21- \\
9.26\end{array}$ & $\begin{array}{l}\text { Panel_II_R1, } \\
\text { Panel_II_R2 }\end{array}$ \\
\hline $3 \mathrm{~A}$ & Tdurum_contig76679_307 & 1326004 & BS00110922_51 & 12813934 & 11.49 & $8 / 8$ & $\begin{array}{l}5.83- \\
9.46\end{array}$ & $\begin{array}{l}\text { Panel_I_R1, } \\
\text { Panel_II_R1, } \\
\text { Panel_II_R2 }\end{array}$ \\
\hline 3B & $A X-111764570$ & 120960387 & IACX5725 & 206195428 & 85.24 & $6 / 10$ & $\begin{array}{l}2.71- \\
5.85\end{array}$ & $\begin{array}{l}\text { Panel_I_R2, } \\
\text { Panel_II_R2 }\end{array}$ \\
\hline $3 \mathrm{D}$ & Excalibur_c18826_452 & 2597023 & AX-109337862 & 92375516 & 89.78 & $4 / 5$ & $\begin{array}{l}2.86- \\
9.81\end{array}$ & $\begin{array}{l}\text { Panel_I_R2, } \\
\text { Panel_II_R1, } \\
\text { Panel_II_R2 }\end{array}$ \\
\hline $4 \mathrm{~A}$ & $A X-111132886$ & 723385268 & & & - & $1 / 1$ & 7.01 & Panel_I_R1 \\
\hline 4B & Excalibur_c32467_676 & 534735432 & Ex_c9296_858 & 589178585 & 54.44 & $4 / 4$ & $\begin{array}{l}5.83- \\
6.65\end{array}$ & Panel_II_R2 \\
\hline $4 \mathrm{D}$ & CAP8_c9110_427 & 121181674 & Excalibur_c38704_1423 & 503621477 & 382.44 & $3 / 3$ & $\begin{array}{l}5.80- \\
9.20\end{array}$ & $\begin{array}{l}\text { Panel_I_R1, } \\
\text { Panel_II_R1, } \\
\text { Panel_II_R2 }\end{array}$ \\
\hline $5 \mathrm{~A}$ & wsnp_Ex_c16317_24795290 & 329828930 & BobWhite_c15758_79 & 595372995 & 265.54 & $60 / 60$ & $\begin{array}{l}3.04- \\
13.32\end{array}$ & $\begin{array}{l}\text { Panel_I_R1, } \\
\text { Panel_I_R2, } \\
\text { Panel_II_R1, } \\
\text { Panel_II_R2 }\end{array}$ \\
\hline $5 B$ & Kukri_c35537_464 & 484637902 & BS00022231_51 & 653655623 & 169.02 & $9 / 9$ & $\begin{array}{l}9.26- \\
9.66\end{array}$ & $\begin{array}{l}\text { Panel_I_R1, } \\
\text { Panel_II_R1 }\end{array}$ \\
\hline $5 \mathrm{D}$ & GENE-3318_556 & 426001273 & wsnp_JD_c825_1223424 & 544697840 & 118.70 & $6 / 6$ & $\begin{array}{l}2.90- \\
10.67\end{array}$ & $\begin{array}{l}\text { Panel_IIR2, } \\
\text { Panel_II_R1, } \\
\text { Panel_II_R2 }\end{array}$ \\
\hline $6 \mathrm{~A}$ & AX-94666339 & 616878 & AX-95658689 & 31355419 & 30.74 & $5 / 6$ & $\begin{array}{l}2.89- \\
11.68\end{array}$ & $\begin{array}{l}\text { Panel_I_R2, } \\
\text { Panel_II_R1 }\end{array}$ \\
\hline $6 \mathrm{~B}$ & wsnp_Ex_c9750_16105678 & 613297099 & Ku_c10647_585 & 618668841 & 5.37 & $4 / 8$ & $\begin{array}{l}5.75- \\
5.75\end{array}$ & Panel_II_R2 \\
\hline $6 \mathrm{D}$ & AX-94426737 & 13568801 & AX-109353709 & 23486224 & 9.92 & $3 / 4$ & $\begin{array}{l}2.80- \\
10.04\end{array}$ & $\begin{array}{l}\text { Panel_I_R1, } \\
\text { Panel_I_R2, } \\
\text { Panel_II_R1 }\end{array}$ \\
\hline $7 \mathrm{~A}$ & AX-86179404 & 515126041 & AX-110051209 & 537557064 & 22.43 & $49 / 57$ & $\begin{array}{l}2.71- \\
7.04\end{array}$ & $\begin{array}{l}\text { Panel_I_R1, } \\
\text { Panel_I_R2 }\end{array}$ \\
\hline 7B & Tdurum_contig47317_100 & 675149692 & AX-94482459 & 714012248 & 38.86 & $8 / 9$ & $\begin{array}{l}3.17- \\
9.38\end{array}$ & $\begin{array}{l}\text { Panel_I_R1, } \\
\text { Panel_I_R2, } \\
\text { Panel_II_R1, } \\
\text { Panel_II_R2 }\end{array}$ \\
\hline 7D & AX-110419815 & 110637 & AX-110963548 & 53290532 & 53.18 & $3 / 5$ & $\begin{array}{l}2.89- \\
10.58\end{array}$ & $\begin{array}{l}\text { Panel_I_R1, } \\
\text { Panel_I_R2, } \\
\text { Panel_II_R1 }\end{array}$ \\
\hline
\end{tabular}

Table 2 Haplotype analysis and phenotypic comparison at the $g F H B-5 A$ locus in two panels 


\begin{tabular}{|c|c|c|c|c|}
\hline Population & Block & Position & Haplotype & Alleles \\
\hline \multirow[t]{2}{*}{ Panel I } & \multirow[t]{2}{*}{ Hap_5A1 } & $577.93 \mathrm{Mb}$ & PI_Hap_5A1a & $T T / G G / C C / C C / C C / G G / G G / A A / T T / G G / G G / A A / C C / A A / A A / A A / C C / T T / T T / T T / G G$ \\
\hline & & $588.75 \mathrm{Mb}$ & PI_Hap_5A1b & $G G / A A / T T / T T / G G / A A / C C / G G / C C / A A / A A / G G / G G / G G / G G / G G / T T / C C / C C / A A / A A$ \\
\hline \multirow[t]{6}{*}{ Panel II } & \multirow[t]{2}{*}{ Hap_5A1 } & $331.4 \mathrm{Mb}$ & PlI_Hap_5A1a & $C C / G G / G G / G T / G G / G G / T T / A A / T T / A A / T T / A G / C C / A A / T T / C C / T T$ \\
\hline & & $422.08 \mathrm{Mb}$ & Pll_Hap_5A1b & $A C / G T / A G / G T / A G / A G / T T / A A / C C / G G / C C / A A / C T / G G / C T / A C / C T$ \\
\hline & \multirow[t]{4}{*}{ Hap_5A2 } & $591.16 \mathrm{Mb}$ & PlI_Hap_5A2a & $A A / T T / C C / G G / A G / A G / T T$ \\
\hline & & $595.37 \mathrm{Mb}$ & PII_Hap_5A2b & $A G / C T / C T / A G / A A / G G / C C$ \\
\hline & & - & PII_Hap_5A1a+PII_Hap_5A2a & - \\
\hline & & - & PII_Hap_5A1b+PII_Hap_5A2b & - \\
\hline
\end{tabular}

Different small and capital letters after numbers means the significance $P<0.05$ and $P<0.01$, respectively.

\section{Figures}

\section{Panel_I Panel_II}

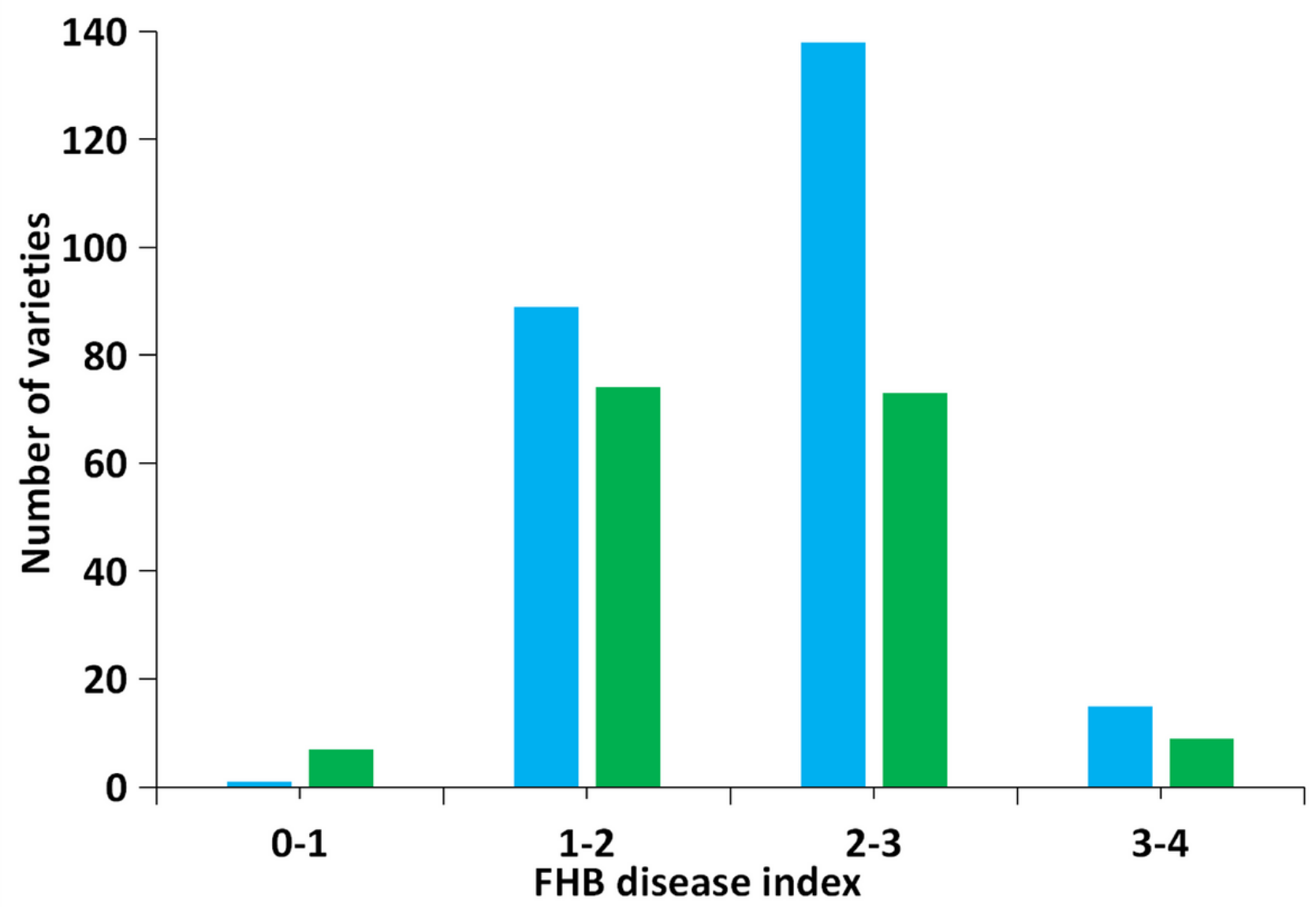

Figure 1

Phenotype distribution of wheat FHB resistance in two panels. (a) The mean value of FHB disease index of two panels. (b) The FHB disease index distribution of two panels. 


$$
\text { a }
$$

Panel_ I R1

b
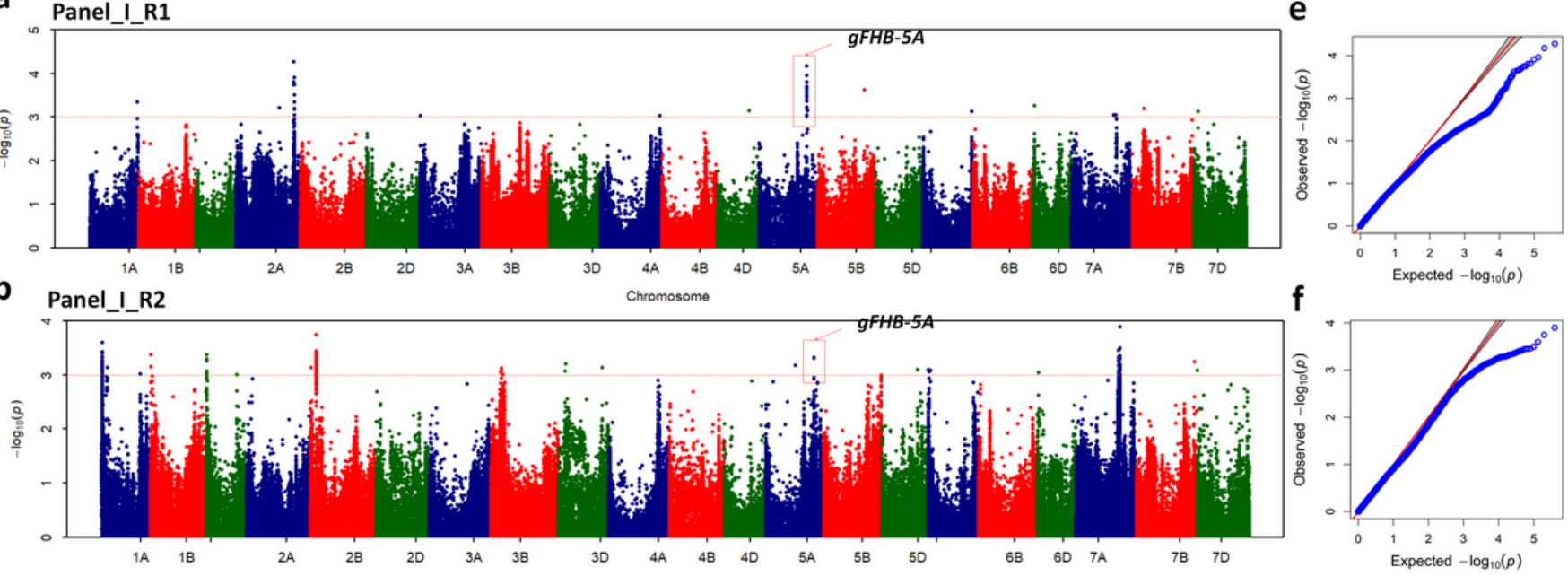

C
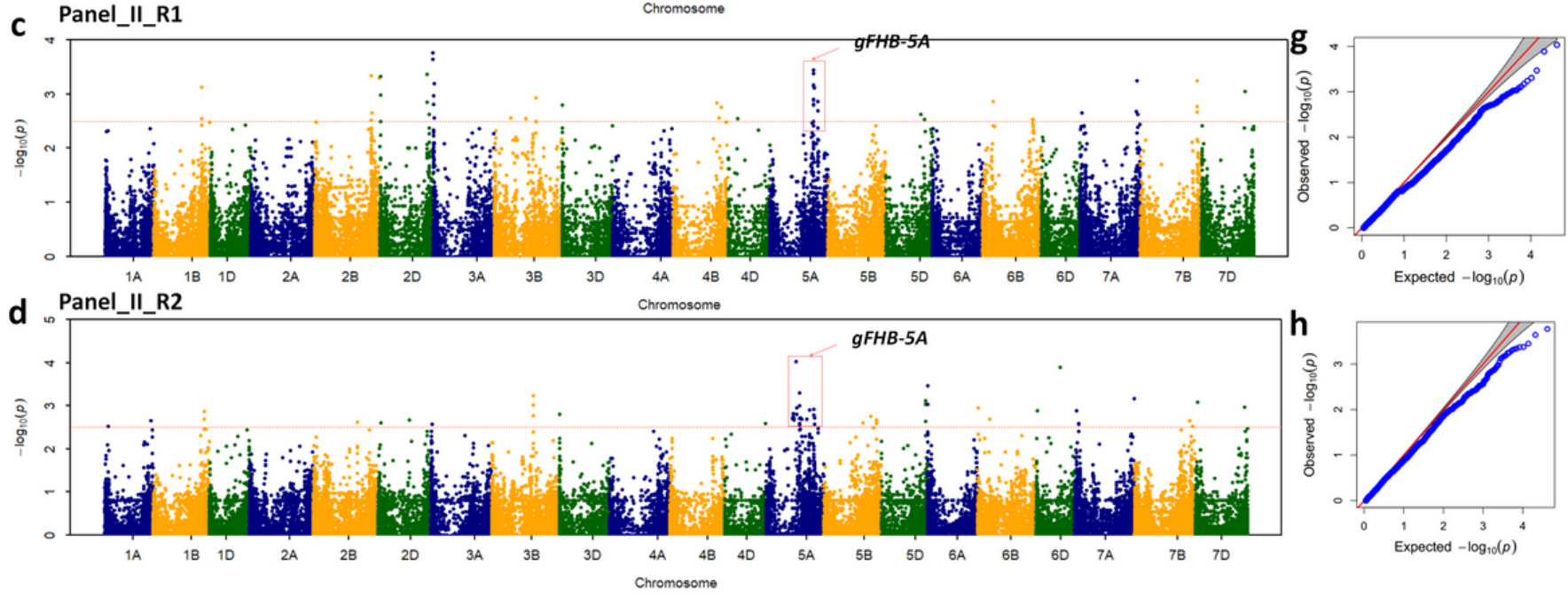

Figure 2

(a-d) Manhattan plots of two replicates of Panel I and Panel II. (e-f) Q-Q plots of two replicates of Panel I and Panel II. 

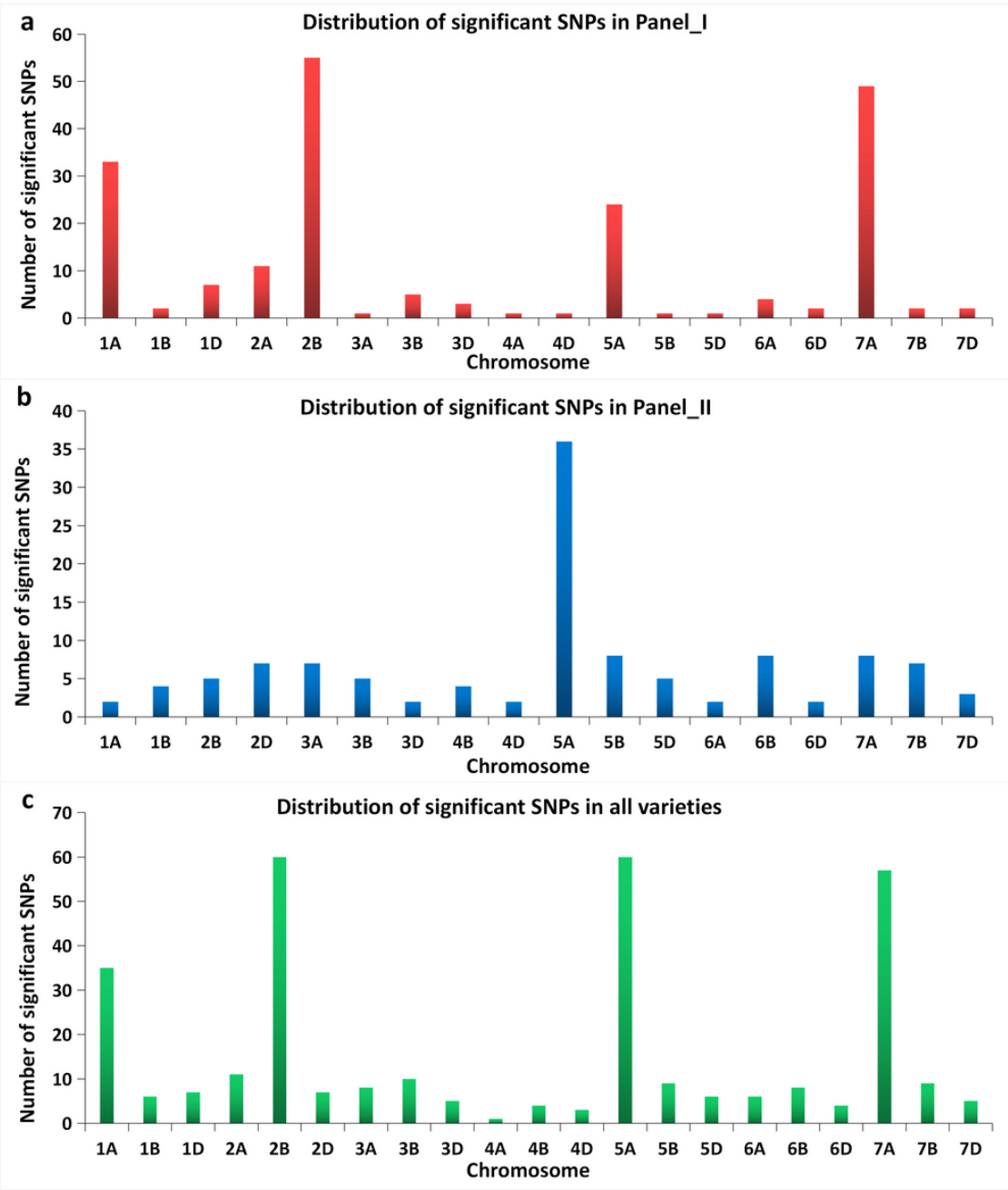

\section{Figure 3}

Number distribution of significant SNPs in Panel I (a), Panel II (b) and all varieties (c). 

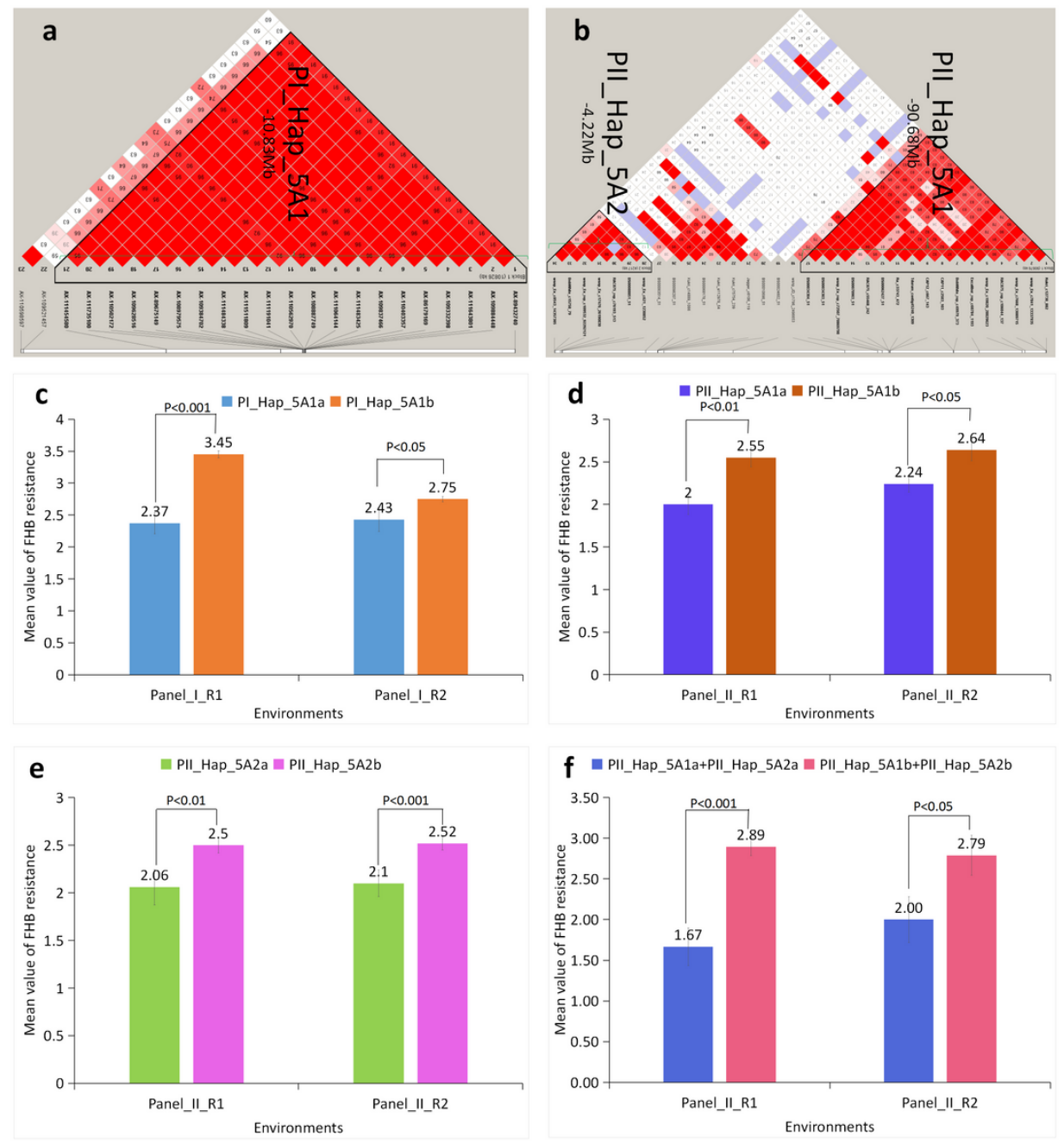

\section{Figure 4}

Haplotype analysis of significant SNPs at gFHB-5A locus. (a) LD heatmap of the significant SNPs in the region of gFHB-5A locus in Panel I and on block were showed in this region. (b) LD heatmap of the significant SNPs in the region of gFHB-5A locus in Panel II and two blocks were showed in this region. (c) Cultivars with haplotype of PI_Hap_5A1a showed stronger FHB resistance than PI_Hap_5A1b. (d) Cultivars with haplotype of PII_Hap_5A1a showed stronger FHB resistance than PII_Hap_5A1b. (e) Cultivars with haplotype of PII_Hap_5A2a showed stronger FHB resistance than PII_Hap_5A2b. (f) Cultivars with polymerized haplotype of PII_Hap_5A1a and PII_Hap_5A2a showed stronger FHB resistance than PII_Hap_5A1b and PII_Hap_5A2b.
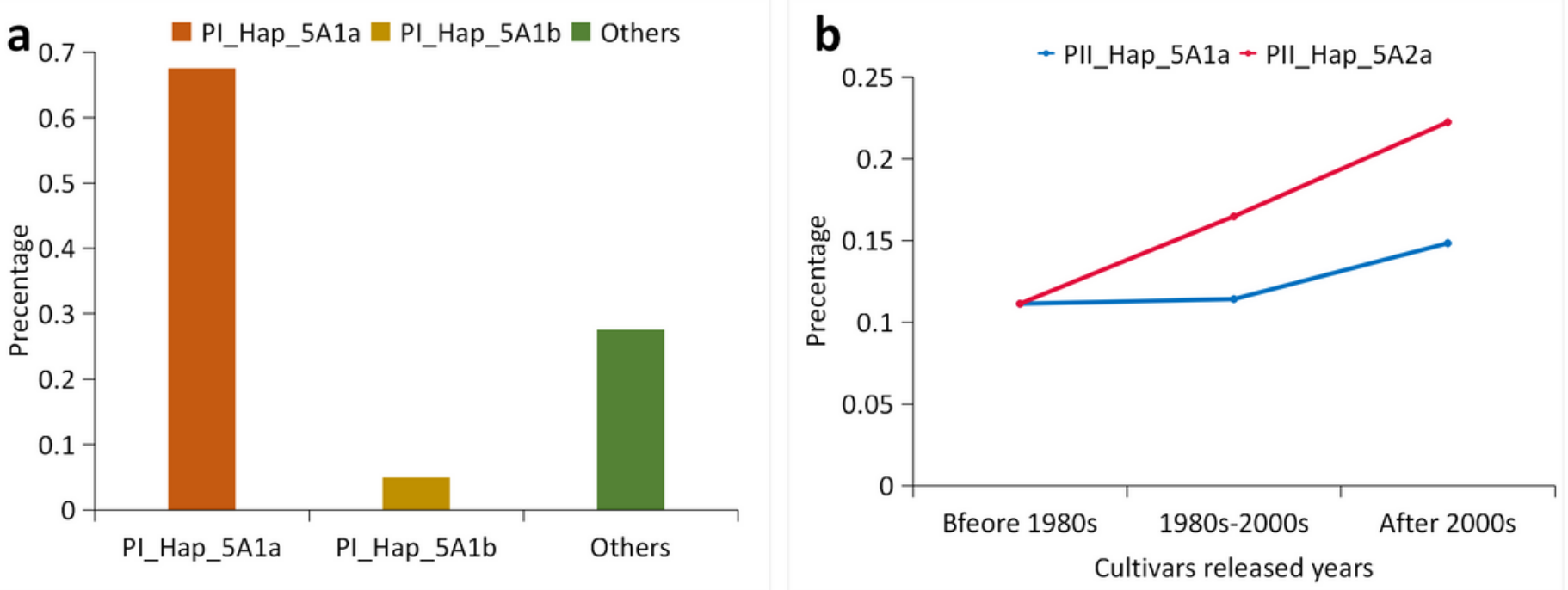
Figure 5

(a) Percentages of superior alleles at the gFHB-5A locus in Panel I. (b) Percentages of superior alleles at the gFHB-5A locus in Panel II that cultivars released before 1980s, 1980s-2000s and after 2000s.

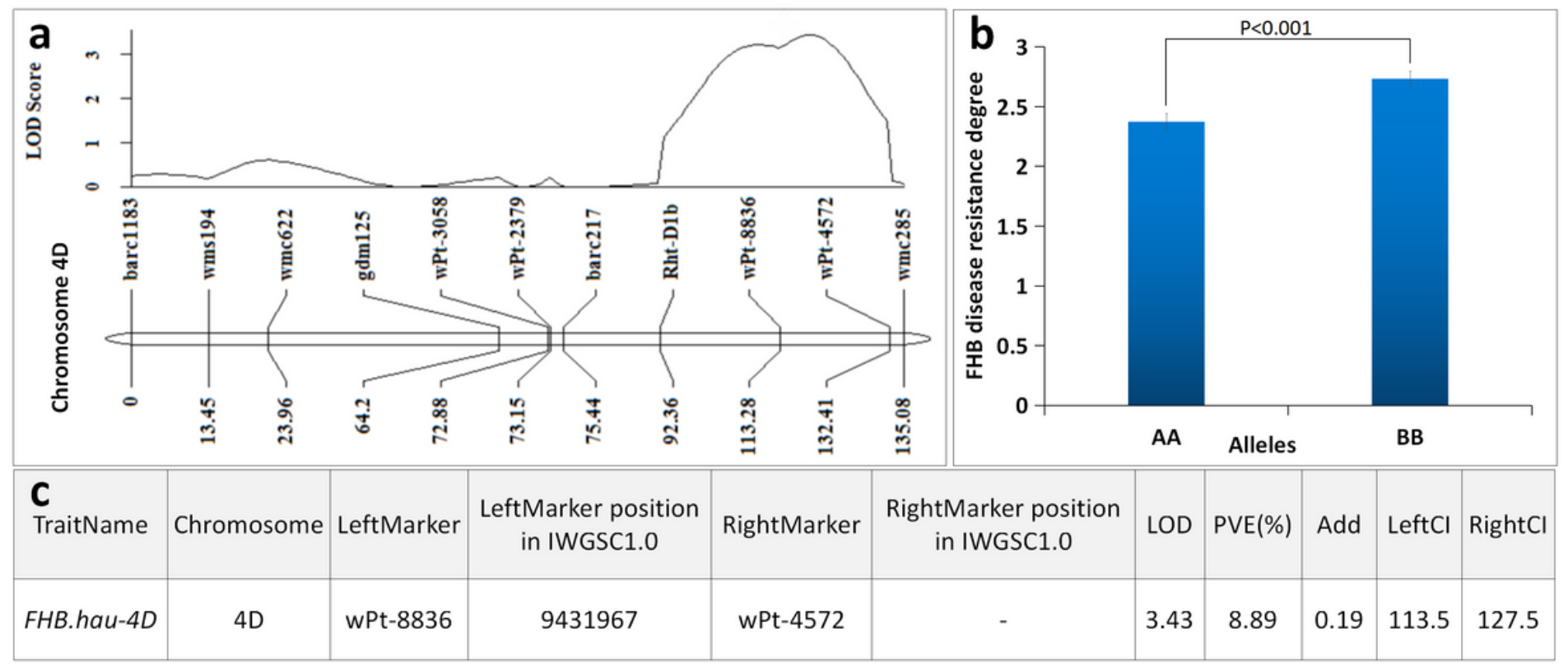

Figure 6

(a) LOD contours for FHB QTL on chromosomes 4D identified in the UC1110 × PI610750 population. (b) FHB resistance comparison of different alleles at the FHB.hau-4D locus. (c) QTL for FHB resistance in the UC1110 × PI610750 population.

\section{Supplementary Files}

This is a list of supplementary files associated with this preprint. Click to download.

- SupplementalTables.xls 